\title{
Temporal multi-omics identifies LRG1 as a vascular niche instructor of metastatic colonization
}

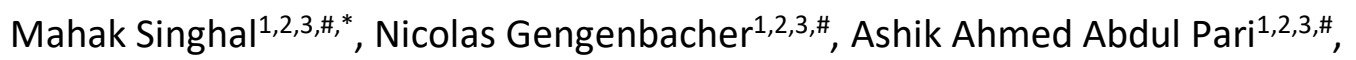 \\ Miki Kamiyama $^{1,2}$, Ling Hai ${ }^{4}$, Bianca Kuhn ${ }^{3,5}$, David M. Kallenberg ${ }^{6}$, Eva Besemfelder ${ }^{1}$, \\ Barbara Leuchs ${ }^{7}$, Carolin Mogler ${ }^{8}$, Jeroen Krijgsveld ${ }^{5}$, Matthias Schlesner ${ }^{4}$, Junhao $\mathrm{Hu}^{9}$, \\ Stephen E. Moss ${ }^{6}$, John Greenwood ${ }^{6}$, Hellmut G. Augustin ${ }^{1,2,10,{ }^{*}}$
}

\begin{abstract}
${ }^{1}$ Division of Vascular Oncology and Metastasis, German Cancer Research Center (DKFZ-ZMBH Alliance), Heidelberg, Germany. ${ }^{2}$ Department of Vascular Biology and Tumor Angiogenesis, European Center for Angioscience (ECAS), Medical Faculty Mannheim, Heidelberg University, Mannheim, Germany. ${ }^{3}$ Faculty of Biosciences, Heidelberg University, Germany. ${ }^{4}$ Junior Group Bioinformatics and Omics Data Analytics, German Cancer Research Center (DKFZ), Heidelberg, Germany. ${ }^{5}$ Divison of Proteomics of Stem Cells and Cancer, German Cancer Research Center (DKFZ), Heidelberg, Germany. ${ }^{6}$ Department of Cell Biology, UCL Institute of Ophthalmology, London, United Kingdom. ${ }^{7}$ Vector Development \& Production Unit, German Cancer Research Center (DKFZ), Heidelberg, Germany. ${ }^{8}$ Institute of Pathology, TUM School of Medicine, Munich, Germany. ${ }^{9}$ Interdisciplinary Research Center on Biology and Chemistry, Shanghai Institute of Organic Chemistry, Chinese Academy of Sciences, Shanghai, China. ${ }^{10}$ German Cancer Consortium, Heidelberg, Germany.
\end{abstract}

\#equally contributing first authors; ${ }^{*}$ Corresponding authors

One sentence summary: A systems-biology endothelial cell screen has established the vascular systems map of early metastatic colonization and identified the TGF $\beta$ pathway specifier as therapeutic target of metastasis.

Short title: $\quad$ LRG1 facilitates metastatic colonization

Keywords: metastasis, vascular niche, angiocrine factors

Word count: $\quad 2,190$

Word count abstract: $\quad 208$

Address correspondence to:

Dr. Hellmut G. Augustin

European Center for Angioscience (ECAS), Medical Faculty Mannheim, Heidelberg University, and German Cancer Research Center (DKFZ-ZMBH Alliance) Im Neuenheimer Feld 280, 69120 Heidelberg, Germany

Phone: +49-6221-421500

Fax: +49-6221-421515

Email: augustin@angiogenese.de 
Metastasis is the primary cause of cancer-related mortality and the mechanistically least well understood step of the tumour progression cascade ${ }^{1-3}$. Tumour cell interactions with cells of the vessel wall are decisive and rate-limiting for metastasis ${ }^{4-6}$. The past decade has witnessed a fundamental change of paradigm from blood vessel wall-lining endothelial cells (EC) being conceived as merely supportive of angiogenesis to an active gatekeeper and modulator of the tumour microenvironment ${ }^{7-12}$. The molecular nature of this crosstalk is beyond candidate gene approaches hitherto poorly understood. Employing surgical models of lung metastasis in temporal systems biology-based screens, we show here that primary tumours systemically reprogram the body's vascular endothelium to perturb homeostasis and to precondition the vascular niche for metastatic colonization. The vasculature with its enormous surface thereby serves as amplifier of tumour-induced instructive signals. The combined endothelial transcriptomic and serum proteomic screen identified the TGF $\beta$ pathway signalling specifier LRG1 as an early instructor of metastatic colonization. Systemic upregulation of LRG1 promoted metastasis by increasing the number of prometastatic NG2+ perivascular cells. In turn, adjuvant LRG1 inhibition in primary tumour-resected mice delayed metastatic growth and increased overall survival. The study has thereby established the systems map of early primary tumour-induced vascular changes and identified LRG1 as a therapeutic target for metastasis.

In order to identify molecular changes of EC in the premetastatic and metastatic niche in an unbiased systems biology approach, we employed surgical metastasis models ${ }^{13}$ and transcriptionally profiled target organ EC over time. A primary screen was performed by subcutaneously inoculating lung metastasising tumour cells (Lewis Lung Carcinoma, LLC) in $\mathrm{C} 57 \mathrm{BL} / 6 \mathrm{~N}$ mice and analysing lung EC at sequential stages of tumour progression, including control (d0), small primary tumour-bearing (d15), 1 wk post-primary tumour resection (d22), and metastasis-bearing (d36) (Fig. 1a). Lung EC were isolated in high purity and used for global transcriptomic profiling (Extended Data Fig. 1a-c). Differential gene expression analysis revealed transcriptional activation of EC upon disease progression (Fig. 1b-d, Extended Data Fig. 1d). Most-significantly altered genes at d15 and d36 were related to protein secretion, inflammatory responses, hypoxia, and cellular proliferation (Fig. 1e, Extended Data Fig. 2a). The presence of a primary tumour evoked a systemic inflammation ${ }^{14,15}$, as evidenced by an inflammatory transcriptomic signature of lung EC (Fig. 1f, g). Concomitantly, a strong immune cell infiltration, particularly of myeloid cells, was observed in d15 lung tissue as compared to d0 (Fig. 1h, Extended Data Fig. 2b, 3a-d). A sharp decline in the expression of inflammatory genes and corresponding infiltrating immune cells was observed at d22 (Fig. 1h, Extended Data Fig. 2a, b, 3a-d), suggesting subsided systemic inflammation following primary tumour resection. Hence, the employed metastasis model truthfully captured the tumour cell-driven systemic alterations including initial myelopoiesis during primary tumour growth, rapid restoration of homeostasis following tumour resection, and finally myeloid cell expansion upon metastatic colonization. Surprisingly though, the immune cell infiltration in d 36 lung tissue was mostly restricted to the adjacent normal tissue rather than the metastatic nodules (Fig. 1h, Extended Data Fig. 2b). Taken together, the data emphasize that the vascular and 
immune compartments within a metastatic organ exhibited a defined temporal signature that mirrors the kinetics of disease progression. Further comparative gene ontology analyses of disease and bio-functions not only supported the immune-phenotyping data but additionally identified disease stage-specific regulation of neovascularization-, cell viability- and metastasis-related gene sets (Fig. 2a).

Zooming-in on genes involved in EC development bio-function, $\operatorname{Lrg} 1$ was identified as one of the most differentially expressed EC-specific genes (Fig. 2b, c, Extended Data Fig. 4a). Lrg1 expression closely reflected the temporal pattern of systemic inflammation, thereby classifying $\operatorname{Lrg} 1$ as an immediate endothelial response gene to tumour challenge. LRG1, Leucine-rich alpha-2-glycoprotein 1, was reported to modulate endothelial TGF $\beta$-signalling ${ }^{16}$. Mechanistically, in the presence of TGF $\beta 1$, LRG1 interacts with the accessory receptor Endoglin, thereby switching the EC phenotype from quiescence-mediating ALK5 signalling to activation-inducing ALK1 signalling ${ }^{16}$. Indeed, upstream regulator analysis of the RNA-seq data revealed TGF $\beta$ as a positively-correlated signalling effector as well as the enrichment of subsequent downstream signalling cascades during metastatic progression (Extended Data Fig. 5a-c). Altered TGF $\beta$-signalling and overall activation of lung endothelium suggested a counter-regulation of the recently described vascular maturation program ${ }^{17}$.

Endothelial STAT3 signalling has been described to actively orchestrate EC responses to inflammation and during metastasis ${ }^{11,18,19}$. Concurrently, we found STAT3 signalling enriched in a disease stage-specific manner in lung EC (Fig. 2d, Extended Data Fig. 2a). To investigate whether STAT3 transcriptionally regulates Lrg1 expression, we employed EC-specific genetic deletion of Stat3 (Extended Data Fig. 6a). Indeed, Stat3 deletion strongly abrogated Lrg1 expression in lung EC isolated from tumour-bearing mice (Fig. 2e). Further, primary tumour experiments in immunocompromised NSG mice manifested reduced levels of $\operatorname{Lrg} 1$ as compared to immunocompetent C57BL/6N mice (Extended Data Fig. 6b). Additionally, tumour cell-derived factors failed to directly induce $\operatorname{Lrg} 1$ expression in mouse lung EC in in vitro Boyden chamber-based experiments (Extended Data Fig. 6c), thereby establishing LRG1 as an endothelial-response factor to tumour-induced systemic inflammation, but not directly tumour cell-derived factors.

We next performed proteomic analyses of serum specimens at sequential stages of LLC tumour progression. Consistent with the transcriptomic screen, LRG1 was one of the most abundant proteins differentially upregulated in $\mathrm{d} 15$ serum as compared to $\mathrm{d} 0$ specimens (Fig. 2f). Supporting the lung EC bulk RNA-seq data, the serum levels of circulating LRG1 closely reflected the temporal pattern of disease progression (Fig. 2g, Extended Data Fig. 7a).

To confirm the findings of the LLC screen in a second, less reductionist tumour model, we orthotopically implanted small bio-banked MMTV-PyMT breast tumour fragments in the mammary fat pads of syngeneic FVB/N mice (Extended Data Fig. 8a), and traced spontaneous metastasis. Similar to the LLC model, Lrg1 was upregulated in lung EC and in serum during metastatic progression in the MMTV-PyMT model (Extended Data Fig. 8b, c). Correspondingly, a meta-analysis of several retrospective clinical studies ${ }^{20-24}$ revealed an upregulation of serum 
120 LRG1 levels for different human cancer entities as compared to corresponding cohorts of

121 healthy volunteers, including colorectal, gastric, lung, ovarian, and pancreatic tumours (Fig.

122 2h). These data underline the systemic regulation of LRG1 during metastatic progression.

123 To determine the primary source of circulating LRG1, we compared Lrg1 expression amongst in vitro-cultured LLC cells, primary tumours, and d15 lung tissue. While LLC cells did not express Lrg1, small levels of $\operatorname{Lrg} 1$ were detectable in the primary tumour (Fig. 3a). Notably however, lung tissue displayed substantially stronger Lrg1 expression as primary tumour tissue (Fig. 3a). To further dissect the cellular source of Lrg1, we isolated EC, leukocytes, and CD $31^{-C D} 45^{-}$cells from both, primary tumour and lung tissue. $\operatorname{Lrg} 1$ expression was enriched in the EC population (Fig. 3b, Extended Data Fig. 9a). Nevertheless, infiltrating leukocytes did express detectable levels of $\operatorname{Lrg} 1$ in primary tumours (Fig. 3b). Next, to investigate the role of leukocyte-derived LRG1 in tumour progression, bone marrow (BM) chimeric mice were generated with either WT or Lrg1-KO BM cells (Extended Data Fig. 9b-d). Lack of leukocytederived LRG1 neither affected primary tumour vasculature (Extended Data Fig. 9e, f), nor did it impact overall survival of mice when compared to the WT BM-chimeras (Fig. 3c). Taken together, EC appear to represent the major cellular source of LRG1, and leukocyte-derived LRG1 is largely-dispensable during metastasis.

To gain insights into EC transcriptomic heterogeneity and to map Lrg1 expression across EC subpopulations, we conducted single-cell RNA sequencing of lung EC isolated at sequential stages of tumour progression. The cellular heterogeneity was investigated both, within and between the samples by applying uniform manifold approximation and projection (UMAP) and graph-based clustering. Following biologically-supervised filtering (Extended Data Fig. 10a), 8,512 cells were annotated as capillary (sub-cluster I/II), arterial, venous, and cycling populations based on the top 10 differentially-expressed genes in each cluster (Fig. 3d, e). The cluster annotation was in line with the current knowledge of prominent EC signalling families including Vegf-Vegfr, Ang-Tie, and Notch (Extended Data Fig. 10b), and corroborated with recently published single-cell data of homeostatic brain and lung $\mathrm{EC}^{25}$. Unexpectedly, the clustering of lung EC remained unaffected during metastasis progression (Extended Data Fig. 10c). Additionally, there were no overt changes in the distribution of cells amongst the clusters (Extended Data Fig. 10d), thereby negating any major restructuring of the vascular hierarchical network as metastatic disease progressed.

Approximately $60 \%$ of venous EC were found positive for $\operatorname{Lrg} 1$ expression (Fig. 3f), attributing to the fact that LRG1 was initially identified as a marker for high-endothelial venules ${ }^{26}$. Yet, $\operatorname{Lrg} 1^{+}$venous cells constituted merely $20 \%$ of total $\operatorname{Lrg} 1^{+}$cells, while the remaining $80 \%$ of $\operatorname{Lrg} 1^{+}$cells were uniformly dispersed amongst the other EC clusters (Fig. $3 \mathrm{~g}$ ). Whilst the frequency of cells expressing $\operatorname{Lrg} 1$ ( $\log _{2}$-normalized expression $>0$ ) remained largely unchanged between the samples, d15 and d36 witnessed a much higher fraction of total cells with elevated levels of Lrg1 expression (Fig. 3h). In concordance with the bulk RNA-seq data, the single-cell data highlighted a systemic upregulation of $\operatorname{Lrg} 1$ expression throughout all lung EC in a tumour stage-specific pattern. Additionally, we examined Lrg1 expression levels in different organ EC and found them to be strongly upregulated at d15 across all examined 
161 vascular beds when compared to the resting vasculature (Fig. 3i). The multiorgan increase in

162 Lrg1 expression was reversed by EC-specific deletion of Stat3 (Fig. 3j), highlighting STAT3 as a

163 key transcriptional regulator of systemic EC $\operatorname{Lrg} 1$ expression. It is noteworthy that enhanced

164 Lrg1 expression across multiple vascular beds might have resulted in the observed increase in

165 serum levels of LRG1, thereby indicating that a primary tumour utilizes the large surface of the body's vascular endothelium as an amplifier of tumour-induced systemically acting angiocrine signals.

To dissect the function of LRG1 during metastatic progression, we established a systemic gainof-function (GOF) experiment by ectopically expressing Lrg1 in LLC cells (Extended Data Fig. 11a-c). Mice were subcutaneously implanted with LLC-pLenti or LLC-Lrg1 tumours. Upon attaining an average tumour size of just $50 \mathrm{~mm}^{3}$, they were intravenously injected with melanoma (B16F10) cells (Extended Data Fig. 11d). Mice with systemic upregulation of LRG1 exhibited a strong increase in melanoma lung metastases (Fig. 4a), thereby establishing a prometastatic role of systemic LRG1. Likewise, in an experimental liver metastasis model, intravenous injection of WT31 cells resulted in a higher metastatic incidence in mice with systemic GOF of LRG1 (Extended Data Fig. 11e, f). To further decipher the exact step of the metastatic cascade, LLC-pLenti and LLC-Lrg1 tumours were resected $24 \mathrm{~h}$ after intravenous injection of B16F10 cells (Extended Data Fig. 12a). There were no differences observed between the two groups suggesting that the pro-metastatic effect of systemic LRG1 was rapidly lost upon withdrawal of the source of LRG1 during metastatic colonization (Extended Data Fig. 12b). To conclusively rule any possible direct effect of LRG1 on tumour cell extravasation, mice were preconditioned with a single injection of either LRG1-neutralizing antibody (anti-LRG1) or control-IgG prior to intravenous injection of melanoma cells (Extended Data Fig. 12c). Consistent with the previous results, blocking LRG1 did not affect the extravasation of melanoma cells (Extended Data Fig. 12d). Collectively, systemically elevated levels of LRG1 supported colonization of disseminated tumour cells.

To investigate the functional impact of LRG1 on the metastatic niche, we quantitated different stromal populations in the lung (Extended Data Fig. 13a). Surprisingly, LRG1 neither influenced EC proliferation nor did it affect the infiltration of different immune cells (Fig. 4b, Extended Data Fig. 13b-d), thereby indicating an angiogenesis- and immune-independent role LRG1 during metastasis. Intriguingly, we observed a strong increase in lung perivascular cells with systemic upregulation of LRG1 (Fig. 4c-e). These NG2+ perivascular cells were recently described to establish a conducive metastatic niche and facilitate metastasis ${ }^{27}$. Therefore, the data suggest that EC-derived LRG1 activates perivascular cells to support metastatic colonization.

Lastly, to assess the therapeutic potential of the LRG1-neutralizing antibody $15 \mathrm{C}^{28}$ in clinically relevant settings, we adopted two therapeutic strategies - short-term perioperative and longterm postsurgical adjuvant therapy (Fig. 4f). Perioperative therapy was initiated after LLC tumours had grown to an average size of $150 \mathrm{~mm}^{3}$ and therapy was discontinued 10 days postprimary tumour resection. This short-term treatment had no apparent effect on the primary tumour vasculature (Extended Data Fig. 14a). Yet, perioperative therapy with anti-LRG1 
yielded a significant overall survival advantage (Fig. 4g). To circumvent any effect on primary tumour growth or early steps in the metastatic cascade such as intravasation and extravasation, we next employed an adjuvant therapy approach wherein administration of anti-LRG1 or control-IgG was commenced 1-day post-primary tumour resection until the experimental endpoint. Similar to the perioperative approach, long-term adjuvant therapy prolonged overall survival of mice by 8.5 days, which corresponded to an approximately $40 \%$ improvement over the control-IgG treated group (Fig. 4h). Remarkably, anti-LRG1 as a monotherapy offered a substantial overall survival advantage in a mouse model which has previously been reported to be refractory to anti-VEGF therapy ${ }^{29}$ and in which chemotherapy shows no effect on lung metastatic burden ${ }^{11}$. Overall, neutralizing LRG1 suppressed tumour cell colonization, thereby providing a significant survival benefit in a clinically-relevant therapeutic window.

In summary, exploiting a comparative systems biology approach, the present study captured the temporal evolution of vascular changes in the pre-metastatic and metastatic niches. Indepth bulk RNA-seq analysis of lung EC complemented with serum proteomics served as a versatile tool for the identification of novel angiocrine molecules. Furthermore, transcriptomics at single-cell resolution mapped endothelial heterogeneity and spatial expression of angiocrine instructors in a tumour cell-seeded lung. The single-cell data added another layer of complexity by attributing spatial information, especially about the arteriovenous axis, which would be diluted in bulk RNA-seq analysis. Notably, the high-resolution expression analysis identifying widespread regulation of LRG1 expression throughout the vascular tree supports the notion that the vascular endothelium serves as an amplifier of tumour-induced systemically-acting instructive signals.

The temporal approach with surgical removal of the primary tumour facilitated for the first time to formally discriminate between pre-metastatic and metastatic EC transcriptomic changes. We prototypically datamined for secreted angiocrine factors and identified the TGF $\beta$ pathway specifier LRG1 as an early EC-specific STAT3-dependent responsive signal that was tightly calibrated to the tumour-induced inflammation. Systemic upregulation of LRG1 was dispensable for extravasation but facilitated early-stage colonization of tumour cells at distant metastatic sites. Concomitantly, intervention with anti-LRG1 suppressed metastatic progression in a clinically-relevant adjuvant regimen. Recently, a phase I/Ila clinical trial with Magacizumab, a humanized version of anti-LRG1 employed in this study, has been initiated for patients with neovascular age-related macular degeneration. Our preclinical data firmly support a crucial role of LRG1 in tumour metastasis and warrant further translational studies of LRG1 as a therapeutic target for metastasis. 
Singhal, Gengenbacher, Abdul Pari et al.

\section{References}

1 Altorki, N. K. et al. The lung microenvironment: an important regulator of tumour growth and metastasis. Nat Rev Cancer 19, 9-31, doi:10.1038/s41568-018-0081-9 (2019).

2 Steeg, P. S. Targeting metastasis. Nat Rev Cancer 16, 201-218, doi:10.1038/nrc.2016.25 (2016).

3 Lambert, A. W., Pattabiraman, D. R. \& Weinberg, R. A. Emerging biological principles of metastasis. Cell 168, 670-691, doi:10.1016/j.cell.2016.11.037 (2017).

4 Augustin, H. G. \& Koh, G. Y. Organotypic vasculature: From descriptive heterogeneity to functional pathophysiology. Science 357, doi:10.1126/science.aal2379 (2017).

5 Butler, J. M., Kobayashi, H. \& Rafii, S. Instructive role of the vascular niche in promoting tumour growth and tissue repair by angiocrine factors. Nat Rev Cancer 10, 138-146, doi:10.1038/nrc2791 (2010).

6 Massague, J. \& Obenauf, A. C. Metastatic colonization by circulating tumour cells. Nature 529, 298-306, doi:10.1038/nature17038 (2016).

7 Cao, Z. et al. Molecular checkpoint decisions made by subverted vascular niche transform indolent tumor cells into chemoresistant cancer stem cells. Cancer Cell 31, 110-126, doi:10.1016/j.ccell.2016.11.010 (2017).

8 Esposito, M. et al. Bone vascular niche E-selectin induces mesenchymal-epithelial transition and Wnt activation in cancer cells to promote bone metastasis. Nat Cell Biol 21, 627-639, doi:10.1038/s41556-019-0309-2 (2019).

$9 \mathrm{Hu}, \mathrm{J}$. et al. Endothelial cell-derived angiopoietin-2 controls liver regeneration as a spatiotemporal rheostat. Science 343, 416-419, doi:10.1126/science.1244880 (2014).

10 Lorenz, L. et al. Mechanosensing by beta1 integrin induces angiocrine signals for liver growth and survival. Nature 562, 128-132, doi:10.1038/s41586-018-0522-3 (2018).

11 Srivastava, K. et al. Postsurgical adjuvant tumor therapy by combining anti-angiopoietin2 and metronomic chemotherapy limits metastatic growth. Cancer Cell 26, 880-895, doi:10.1016/j.ccell.2014.11.005 (2014).

12 Wieland, E. et al. Endothelial Notch1 activity facilitates metastasis. Cancer Cell 31, 355367, doi:10.1016/j.ccell.2017.01.007 (2017).

13 Gengenbacher, N., Singhal, M. \& Augustin, H. G. Preclinical mouse solid tumour models: status quo, challenges and perspectives. Nat Rev Cancer 17, 751-765, doi:10.1038/nrc.2017.92 (2017).

14 De Palma, M., Biziato, D. \& Petrova, T. V. Microenvironmental regulation of tumour angiogenesis. Nat Rev Cancer 17, 457-474, doi:10.1038/nrc.2017.51 (2017).

15 Peinado, H. et al. Pre-metastatic niches: organ-specific homes for metastases. Nat Rev Cancer 17, 302-317, doi:10.1038/nrc.2017.6 (2017).

16 Wang, $X$. et al. LRG1 promotes angiogenesis by modulating endothelial TGF-beta signalling. Nature 499, 306-311, doi:10.1038/nature12345 (2013).

17 Schlereth, K. et al. The transcriptomic and epigenetic map of vascular quiescence in the continuous lung endothelium. Elife 7, doi:10.7554/eLife.34423 (2018).

18 Kano, A. et al. Endothelial cells require STAT3 for protection against endotoxin-induced inflammation. J Exp Med 198, 1517-1525, doi:10.1084/jem.20030077 (2003).

$19 \mathrm{Kim}, \mathrm{K}$. J. et al. STAT3 activation in endothelial cells is important for tumor metastasis via increased cell adhesion molecule expression. Oncogene 36, 5445-5459, doi:10.1038/onc.2017.148 (2017).

20 Yamamoto, M. et al. Overexpression of leucine-rich alpha2-glycoprotein-1 is a prognostic marker and enhances tumor migration in gastric cancer. Cancer Sci 108, 2052-2060, doi:10.1111/cas.13329 (2017). 
21 Shinozaki, E. et al. Serum leucine-rich alpha-2-glycoprotein-1 with fucosylated triantennary N-glycan: a novel colorectal cancer marker. BMC Cancer 18, 406, doi:10.1186/s12885-018-4252-6 (2018).

22 Liu, Y. S. et al. Shotgun and targeted proteomics reveal that pre-surgery serum levels of LRG1, SAA, and C4BP may refine prognosis of resected squamous cell lung cancer. $J \mathrm{Mol}$ Cell Biol 4, 344-347, doi:10.1093/jmcb/mjs050 (2012).

23 Furukawa, K. et al. Clinicopathological significance of Leucine-Rich alpha2-Glycoprotein-1 in sera of patients with pancreatic cancer. Pancreas 44, 93-98, doi:10.1097/MPA.0000000000000205 (2015).

24 Andersen, J. D. et al. Leucine-rich alpha-2-glycoprotein-1 is upregulated in sera and tumors of ovarian cancer patients. J Ovarian Res 3, 21, doi:10.1186/1757-2215-3-21 (2010).

25 Vanlandewijck, M. et al. A molecular atlas of cell types and zonation in the brain vasculature. Nature 554, 475-480, doi:10.1038/nature25739 (2018).

26 Saito, K. et al. Gene expression profiling of mucosal addressin cell adhesion molecule-1+ high endothelial venule cells (HEV) and identification of a leucine-rich HEV glycoprotein as a HEV marker. J Immunol 168, 1050-1059 (2002).

27 Murgai, M. et al. KLF4-dependent perivascular cell plasticity mediates pre-metastatic niche formation and metastasis. Nat Med 23, 1176-1190, doi:10.1038/nm.4400 (2017).

28 Moss, S. E. et al. Preclinical development and testing of a therapeutic antibody against LRG1 [abstract]. In: Proc. Am. Assoc. Cancer Res. Ann. Meeting 2018; Cancer Res 78(13 Suppl), Abstract No. 5757 (2018).

29 Shojaei, F. et al. Tumor refractoriness to anti-VEGF treatment is mediated by CD11b+Gr1+ myeloid cells. Nat Biotechnol 25, 911-920, doi:10.1038/nbt1323 (2007). 
311 Acknowledgements: The authors would like to thank Prof. Dr. Jonathan Sleeman (ECAS,

312 Medical Faculty Mannheim, Heidelberg University, Germany) for providing MMTV-PyMT 313 tumours. We thank Prof. Dr. Ralf Adams (Max Planck Institute for Molecular Biomedicine, 314 Muenster, Germany) for providing VECadCre ${ }^{\text {ERT2 }}$ mice. We thank Prof. Dr. Franklin Constantini 315 (Columbia University, New York, NY) for providing Rosa-YFPfl/fl (YFPfl/fl) mice. We thank Prof.

316 Dr. Mathias Heikenwälder (German Cancer Research Center, Heidelberg, Germany) for 317 providing Stat3 ${ }^{\mathrm{fl} / \mathrm{fl}}$ mice. We thank Ignacio Heras Murillo and Dr. Christopher Previti for 318 technical assistance. We are most grateful for the excellent technical support of the Flow 319 Cytometry, the Light Microscopy, the Genomics and Proteomics, the Omics IT and Data 320 Management, and the Laboratory Animal Facilities of the DKFZ and IRCBC.

321 Funding: This work was supported by grants from the Deutsche Forschungsgemeinschaft 322 (DFG) (project C5 within CRC1366 "Vascular control of organ function" [project number 32339404578 to H.G.A.] and projects A2 and Z4 within CRC1324 "Wnt signalling" [project number 324331351713 to H.G.A. and J.K.]); the European Research Council Advanced Grant 325 "AngioMature" [project 787181 to H.G.A.]; and DFG-funded Research Training Group 2099 326 "Hallmarks of Skin Cancer" [project P8 to H.G.A.]. J.H. is supported by the "The Thousand 327 Young Talents Recruitment Program". J.G. and S.E.M. are supported by the Wellcome Trust 328 Investigator Award [206413/B/17/Z] and Medical Research Council UK [MR/N006410/1].

329 Author contributions: MS, NG, AAAP, and HGA conceived and designed the study. MS, NG, 330 AAAP, MK, LH, BK, BL, CM, JK, MSc, and JH performed experiments. DMK, SEM, and JG 331 provided reagents. EB provided technical support. MS, NG, AAAP, and HGA analysed and 332 interpreted data. MS and HGA supervised the project. MS, NG, AAAP, and HGA wrote the 333 manuscript. All authors discussed the results and commented on the manuscript.

334 Competing interests: Authors declare no competing interests.

335 Data and materials availability: NGS data have been deposited in the Gene Expression 336 Omnibus under accession numbers GSE131072 (bulk RNA-seq) and GSE131110 (single-cell 337 RNA-seq). The mass spectrometry data files have been deposited to the ProteomeXchange 338 Consortium under the accession number PXD013978. 
a
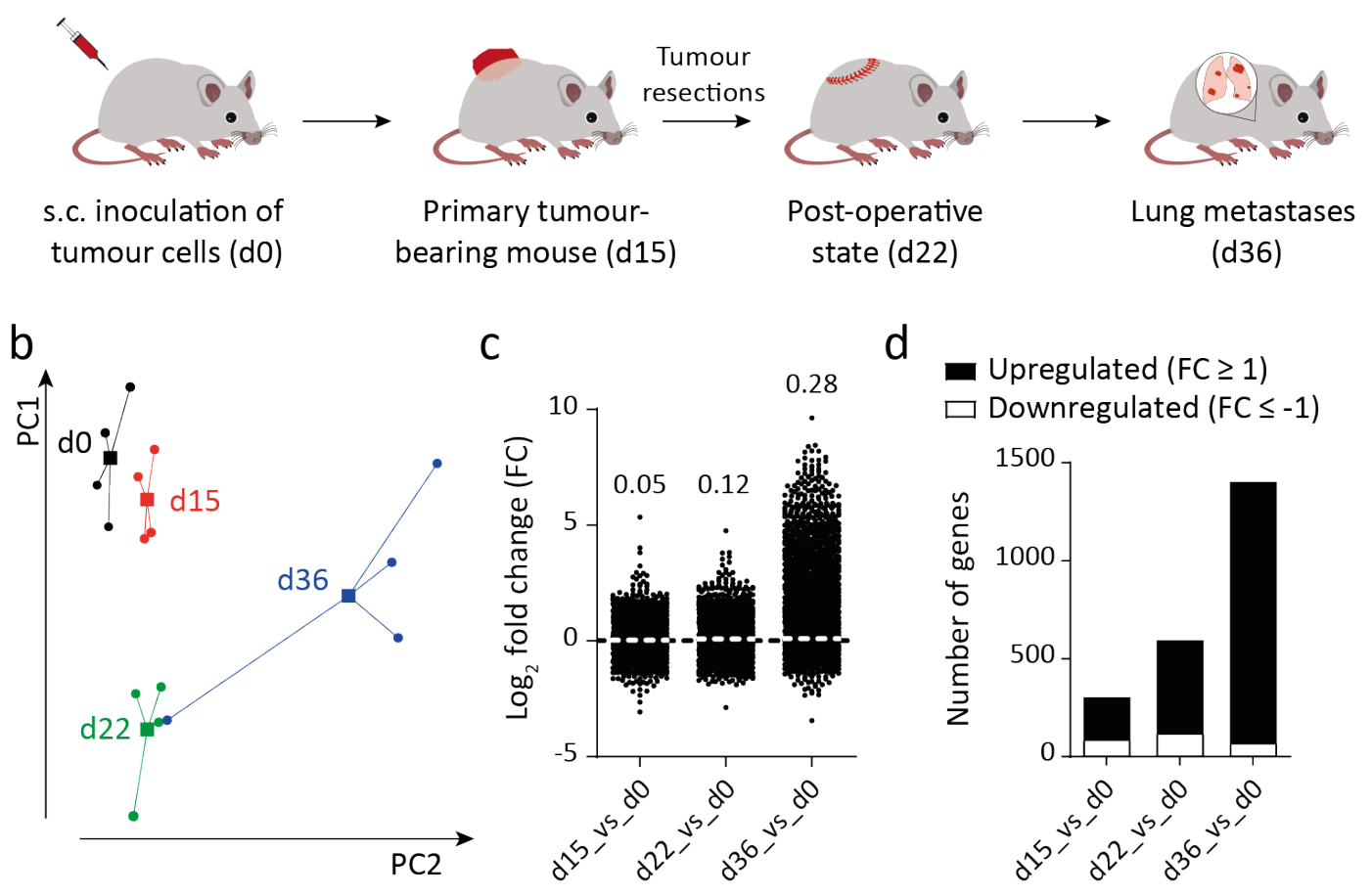

e

Hallmark gene sets (d15_vs_do)

f

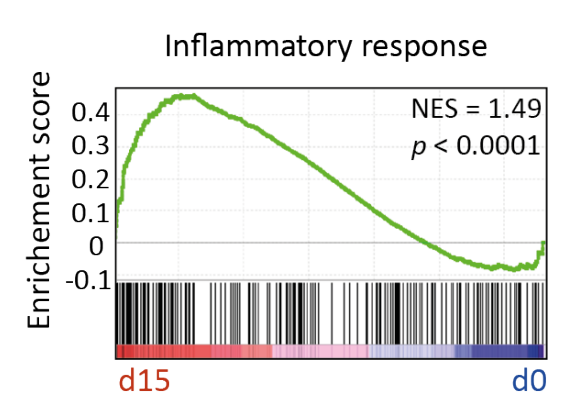

g

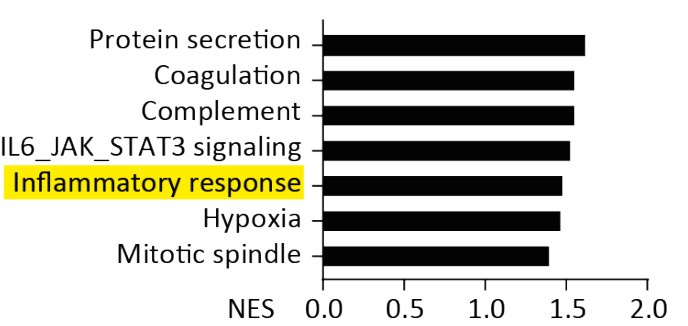

8

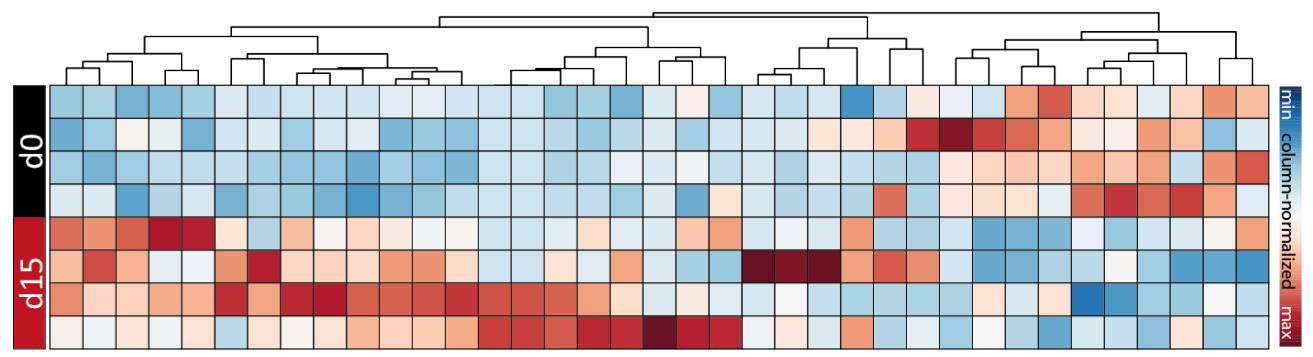

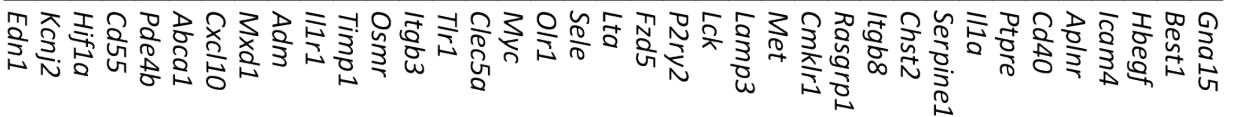

$\mathrm{h}$

do

$\mathrm{d} 15$
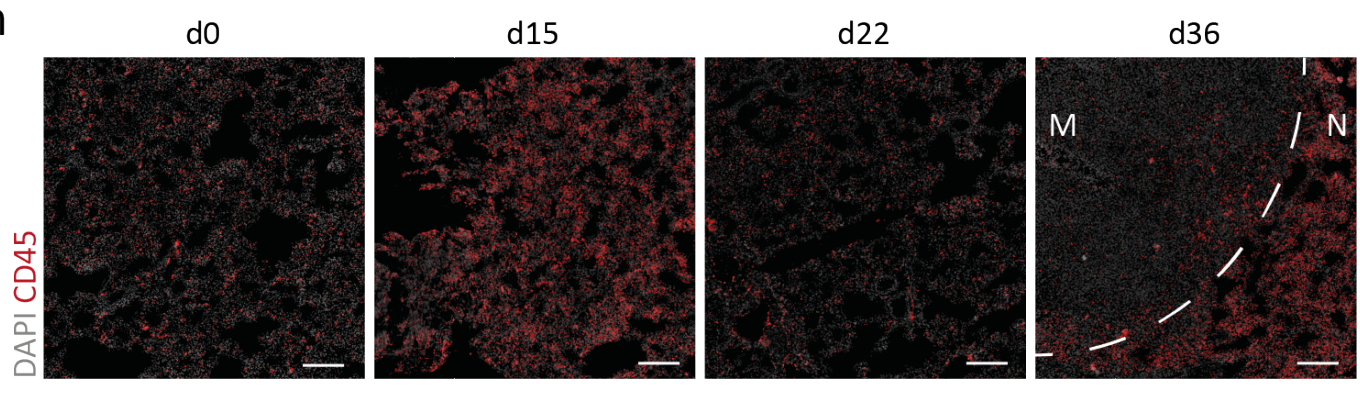
Fig. 1| Transcriptomic evolution of lung EC during metastasis. a, Schematic depiction of LLC spontaneous metastasis model, in which mice develop lung metastases following primary tumour resection. $\mathbf{b}$, Principal component analysis of RNA-seq data of isolated lung EC ( $n=4$ samples for each time point). Circles and squares denote individual samples and centroid of each group, respectively. $c$, Dot plot showing $\log _{2}$ fold change $(F C)$ for genes with $R P K M \geq 1$ in at least one of the samples. The mean FC of all analysed genes is indicated for each comparison. $d$, Bar graph illustrating the number of significantly upregulated $(\uparrow)$ and downregulated $(\downarrow)$ genes in d15 (226 个, $89 \downarrow)$, d22 (480 个, $119 \downarrow)$, and d36 (1329 个, $71 \downarrow)$ lung EC as compared to d0. e, Gene Set Enrichment Analysis (GSEA) comparing $\mathrm{d} 15$ and d0 data sets. $\mathbf{f}$, The inflammatory response gene set was found positively-correlated with $\mathrm{d} 15$ time point. $\mathbf{g}$, Heatmap highlighting genes in the inflammatory response gene set. $\mathbf{h}$, Immunofluorescence images showing infiltrating $\mathrm{CD} 45^{+}$immune cells in the lung tissue. Scale bars $=200 \mu \mathrm{m}$. $\mathrm{M}=$ metastatic nodule; $\mathrm{N}=$ normal adjacent tissue; $\mathrm{NES}=$ normalized enrichment score. 
a

Disease and bio-functions

Endothelial cell development Vascularization Neovascularization Cell viability Metastasis Chemotaxis Leukocyte migration Activation of granulocytes Adhesion of neutrophils Binding of cells Cell rolling of leukocytes go ja ja $42,72 / 2$ $d^{5} \frac{1}{2} \frac{1}{d^{3}}$

d

Hallmark_IL6_JAK_STAT3_signaling
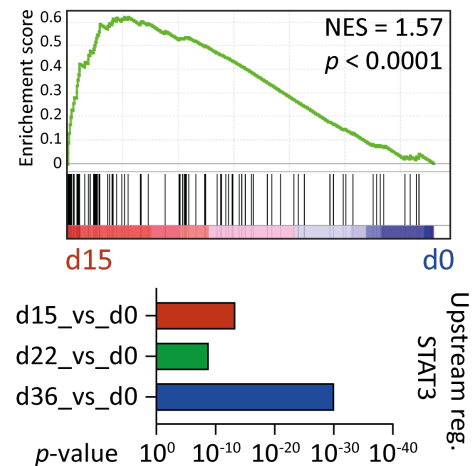

$f$

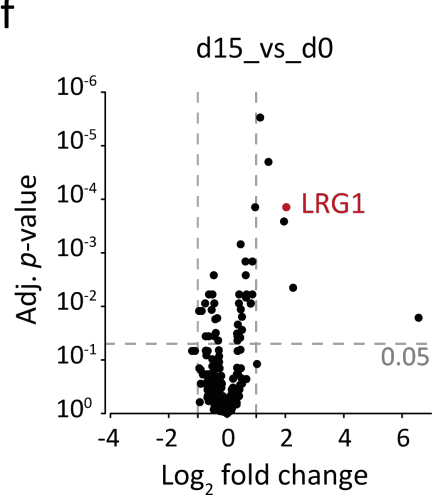

g

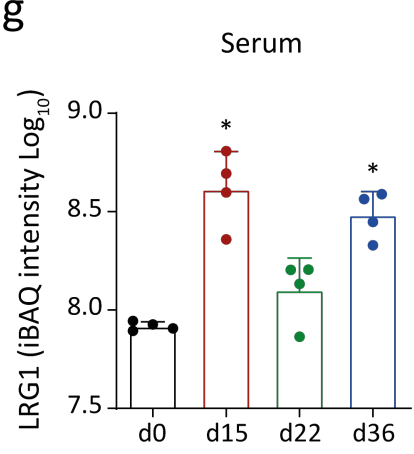

b

e
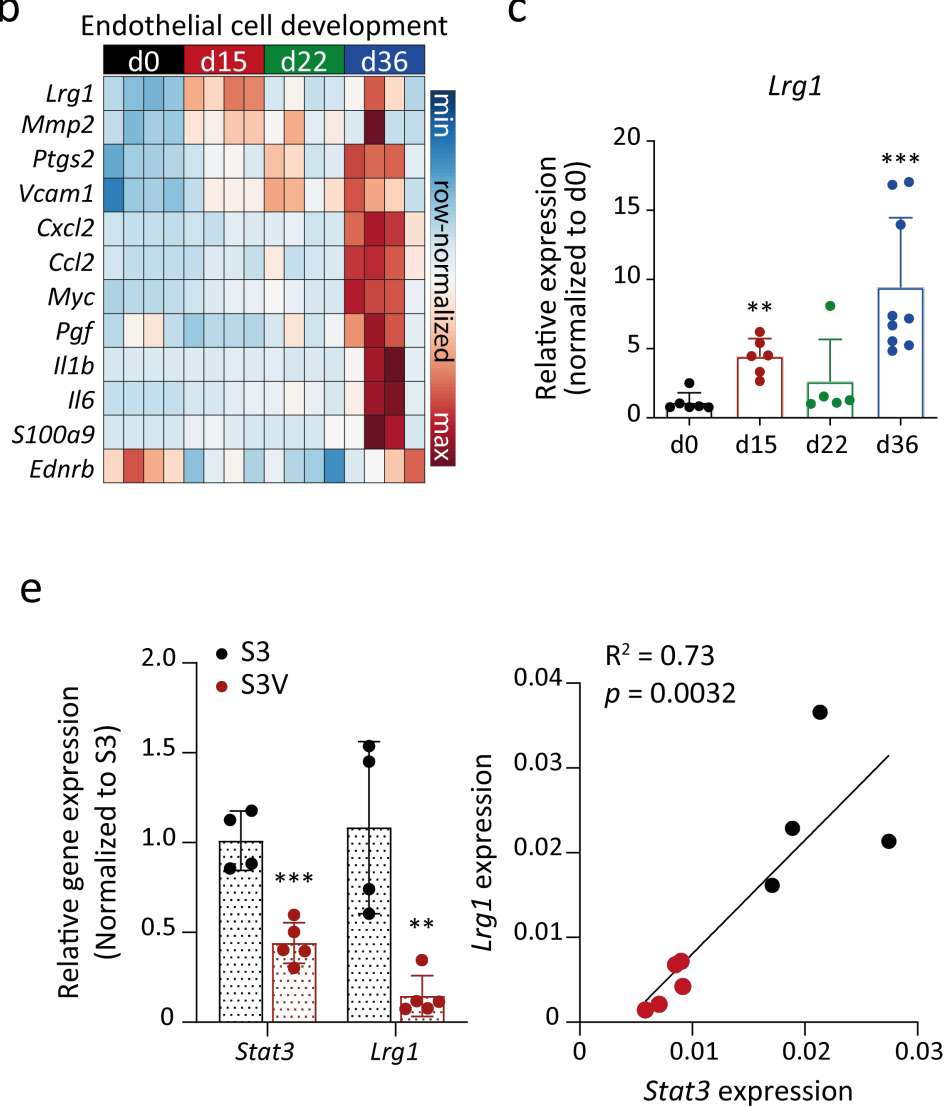

h

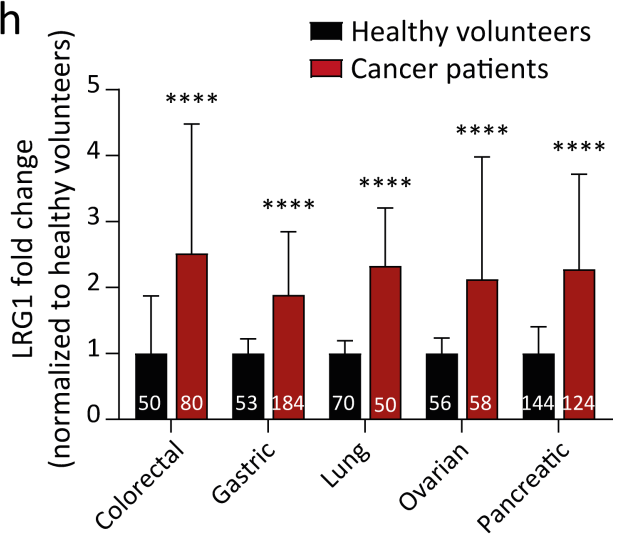


Fig. 2 | LRG1 is systemically elevated during tumour progression. a, Comparison of disease and biofunctions was conducted using Ingenuity Pathway Analysis (IPA). Correlation scores (z-score) are shown for the selected disease and bio-functions. $\mathbf{b}$, Genes involved in the EC development gene set are shown in row-normalized $\log _{2}$-expression values. c, qPCR quantitation of $\operatorname{Lrg} 1$ expression in lung $\mathrm{EC}$ to validate RNA-seq data (mean $\pm \mathrm{SD}, \mathrm{n}=5-9$ mice). ${ }^{* *}, \mathrm{P}<0.01 ; * * *, \mathrm{P}<0.001$ (two-tailed Mann-Whitney $U$ test). d, GSEA plot highlighting enriched IL6_JAK_STAT3 signalling on d15 as compared to d0 (upper panel). IPA analysis revealing STAT3 as an upstream regulator in disease stage-specific pattern. e, On the left, qPCR analysis of Stat3 and Lrg1 expression in lung EC isolated from tumour-bearing Stat $3^{\mathrm{fl} / \mathrm{fl}}(\mathrm{S} 3)$ or Stat $3^{\mathrm{fl} / \mathrm{fl}} \times \mathrm{VECadCre}^{\mathrm{ERT2}}(\mathrm{S} 3 \mathrm{~V})$ mice (mean $\pm \mathrm{SD}, \mathrm{n}=4-5$ mice). **, $\mathrm{P}<0.01 ; * * *, \mathrm{P}<0.001$ (multiple t-tests corrected with the Holm-Sidak method). On the right, Pearson's correlation between Stat 3 and Lrg1 expression. $\mathbf{f}$, Volcano plot displaying FC and adjusted $p$-value for each identified protein in LC-MS analyses. The mean of 4 biological replicates is indicated. $g$, Shown are iBAQ intensities of LRG1 protein in serum samples (mean $\pm S D, n=4$ mice). *, $\mathrm{P}<0.05$ (two-tailed Mann-Whitney $U$ test). $\mathrm{h}, \mathrm{LRG1}$ protein amounts in sera of cancer patients and healthy volunteers were retrieved from previously-published articles (13-17). The bar graph shows relative LRG1 abundance normalized to the corresponding healthy cohort. Data normalization removes differences originating due to varying measurement techniques employed in different studies. The size of each sample cohort is indicated in the graph. ${ }^{* * * *}, \mathrm{P}<0.0001$ (multiple t-tests corrected with the Holm-Sidak method). 
Singhal, Gengenbacher, Abdul Pari et al.

a

b

C
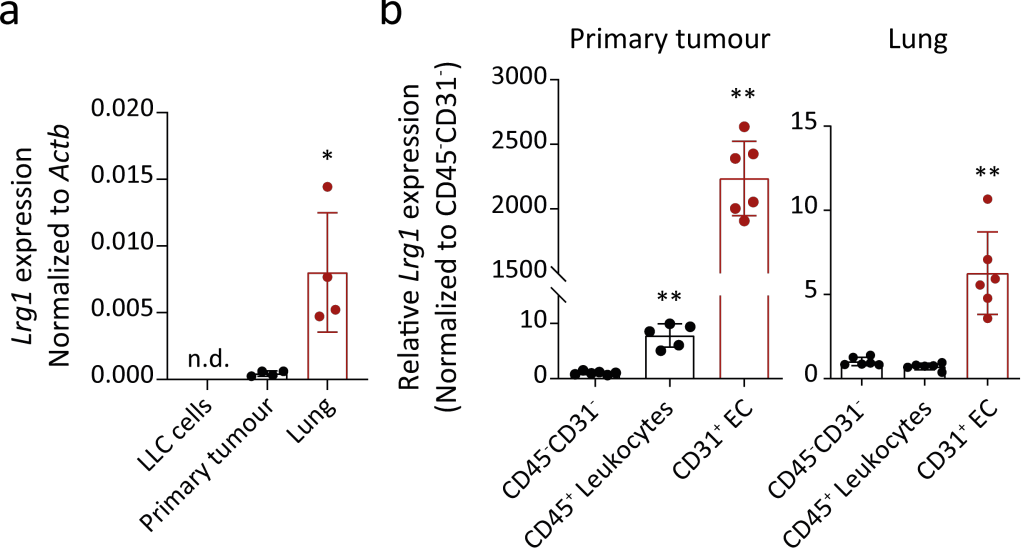
$\begin{array}{ll}\mathrm{ns} & \perp \text { WT BM chimera } \\ \perp \text { Lrg1-KO BM chimera }\end{array}$

d
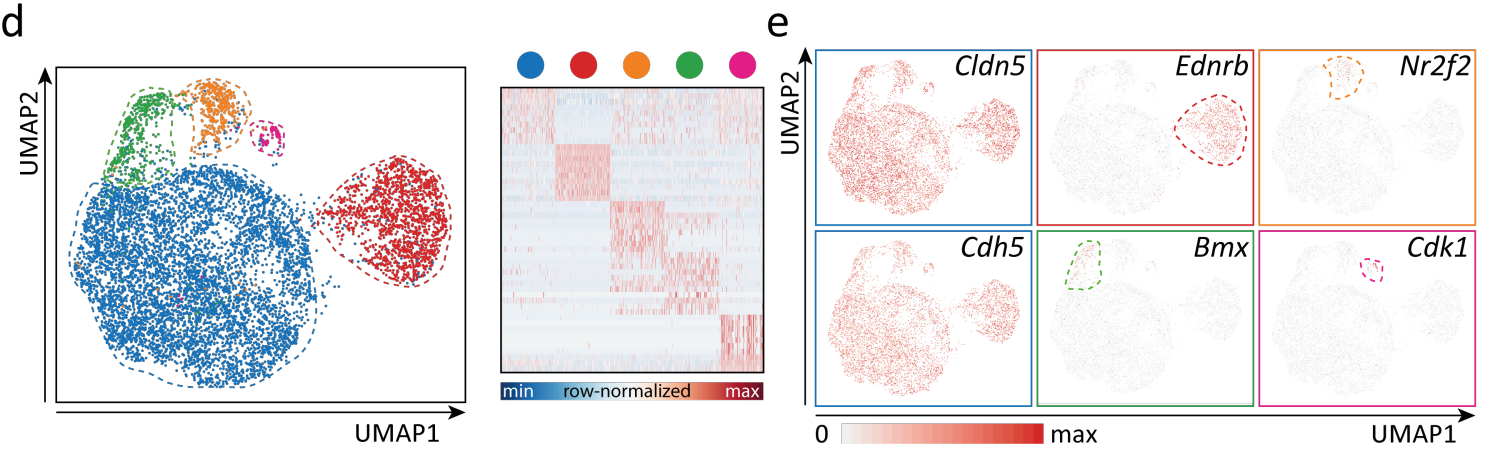

Capillary I $(n=6223) \quad$ Capillary II $(n=1427) \quad$ Venous $(n=383)$

$\mathrm{f}$

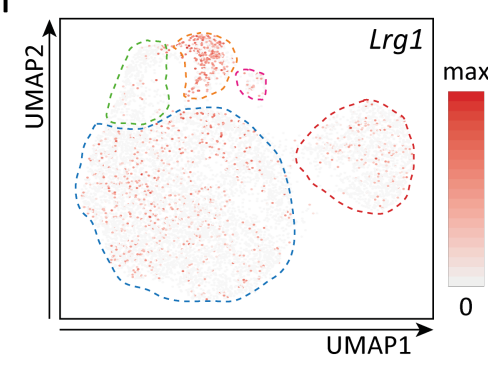

g

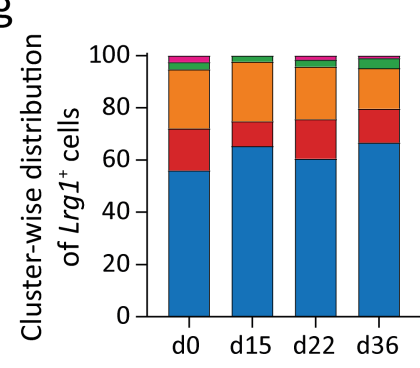

$\mathrm{h}$

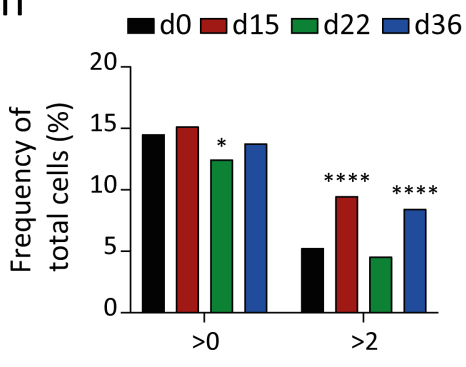

$\log _{2}$ normalized $\operatorname{Lrg} 1$ expression i

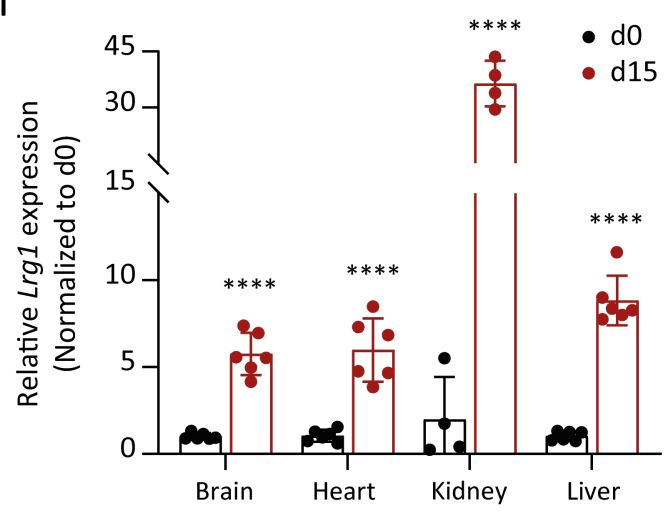

j

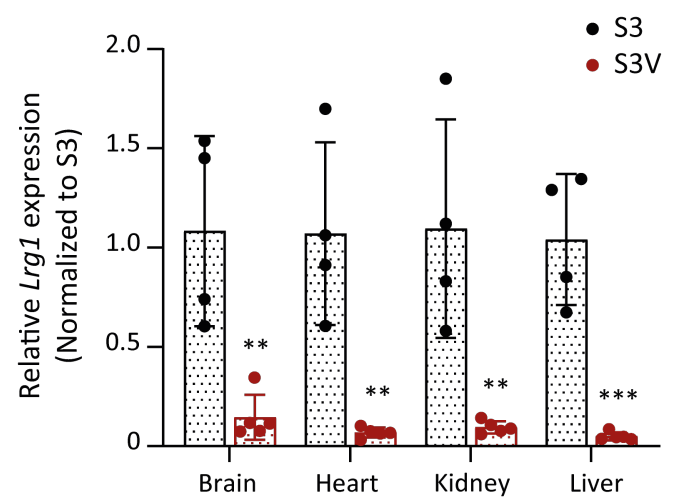


Fig. 3 | Vascular endothelial cells are the major source of LRG1 and serve as a signal amplifier. a, Comparison of Lrg1 expression between in vitro-cultured LLC cells, primary tumour and d15 lung tissue (mean $\pm \mathrm{SD}, \mathrm{n}=4$ mice). ${ }^{*}, \mathrm{P}<0.05$ (two-tailed Mann-Whitney $U$ test). $\mathbf{b}, \mathrm{EC}$, leukocytes, and CD31 ${ }^{-}$CD $45^{-}$cells were isolated from primary tumours and d15 lung tissues. Dot plots show relative Lrg1 expression in EC and leukocytes as compared to CD31 ${ }^{-}$CD45 cells (mean $\pm S D, n=5-6$ mice). **, $\mathrm{P}<0.01$ (two-tailed Mann-Whitney $U$ test). c, LLC tumours were implanted in WT or Lrg1-KO BM chimeras. Kaplan-Meier graph showing overall survival of mice after primary tumour resection $(\mathrm{n}=$ 8-9 mice). The comparison was rendered non-significant (ns) according to Log-rank (Mantel-Cox) test. $\mathbf{d}$, On the left, UMAP visualization of colour-coded clusters of lung EC ( $n=8,512$ cells). On the right, gene signature of the capillary I/II, arterial, venous, and cycling subpopulations based on 10 most-upregulated genes. e, Feature plots indicating enriched genes for each identified subpopulation. EC-specific Cldn5 and Cdh5 were uniformly expressed by all subpopulations. $\mathbf{f}$, Feature plot displaying Lrg1 expression across all analysed lung EC. g, Shown is the cluster-wise spread of Lrg1-expressing cells for each sample. $\mathbf{h}$, The graph highlights the frequency of Lrg1expressing cells ( $\log _{2}$-normalized expression $>0$ or $>2$ ) amongst the total number of cells per sample. *, $\mathrm{P}<0.05 ;{ }^{* * * *}, \mathrm{P}<0.0001$ (two-sided Fischer's exact test). $\mathbf{i}$, Lrg1 expression was analysed in $\mathrm{EC}$ isolated from multiple organs of $\mathrm{d} 0$ and $\mathrm{d} 15$ mice (mean $\pm \mathrm{SD}, \mathrm{n}=4-6$ mice). ${ }^{* * * *}, \mathrm{P}<0.0001$ (twotailed Mann-Whitney $U$ test). $\mathbf{j}$, Lrg1 expression in multiorgan EC isolated from tumour-bearing Stat3 $^{\mathrm{fl} / \mathrm{fl}}(\mathrm{S} 3) \operatorname{or~Stat3}^{\mathrm{fl} / \mathrm{fl}} \mathrm{XVECadCre}{ }^{\mathrm{ERT2}}$ (S3V) mice (mean $\pm \mathrm{SD}, \mathrm{n}=4-5$ mice). ${ }^{* *}, \mathrm{P}<0.01 ; * * *, \mathrm{P}<0.001$ (multiple t-tests corrected with the Holm-Sidak method). n.d. = non-detectable. 
a

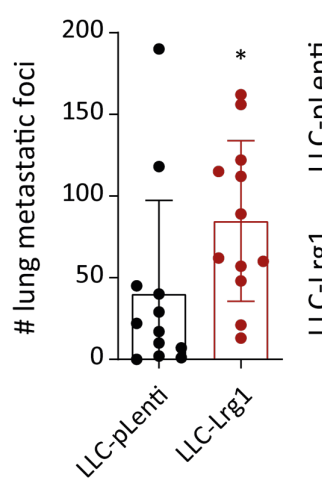

d
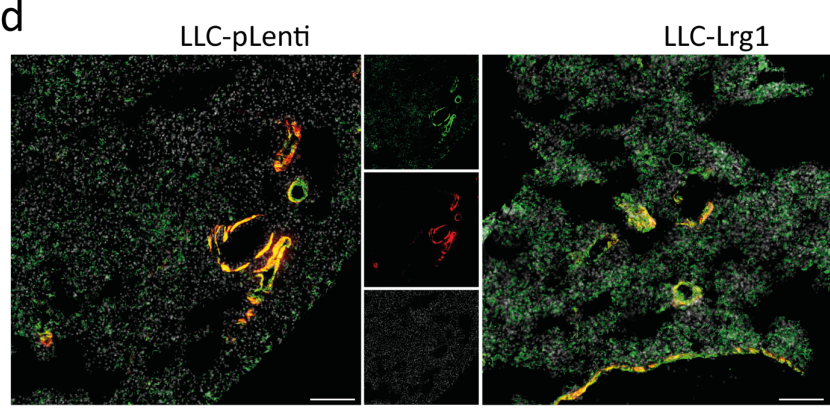

Desmin / $\alpha$ SMA / DAP

f

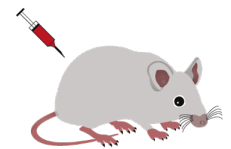

s.c. inoculation of LLC cells

Perioperative Adjuvant

g

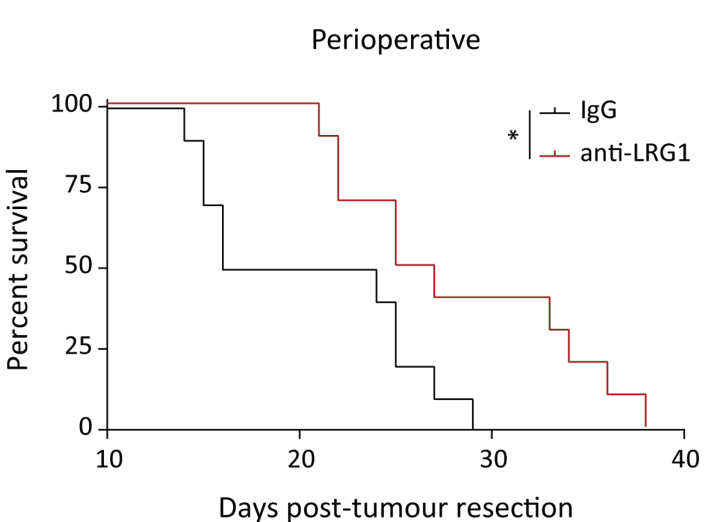

h b

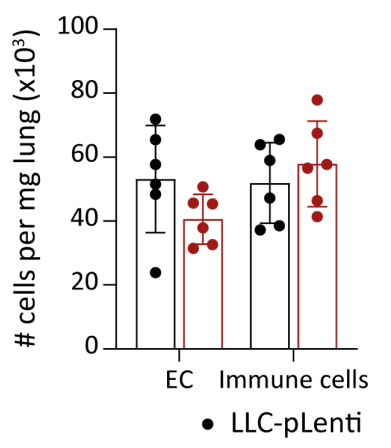

C

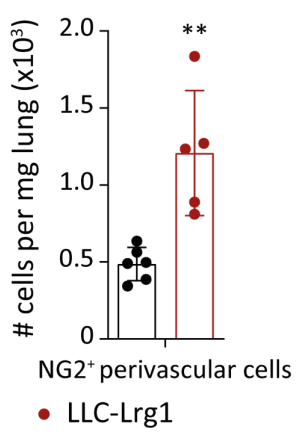

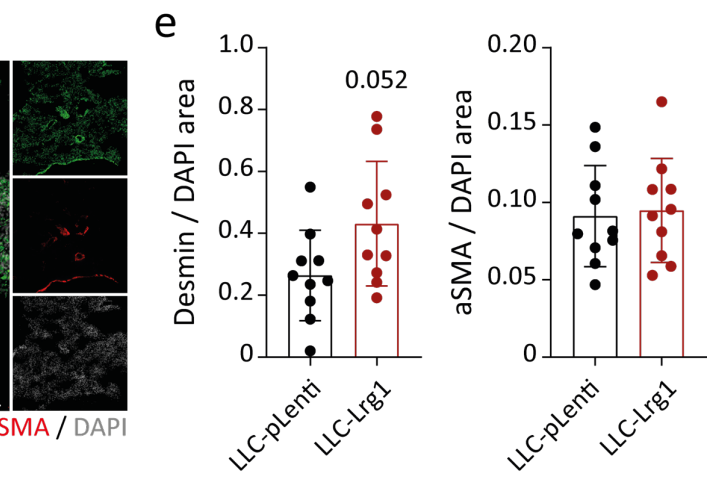

Adjuvant

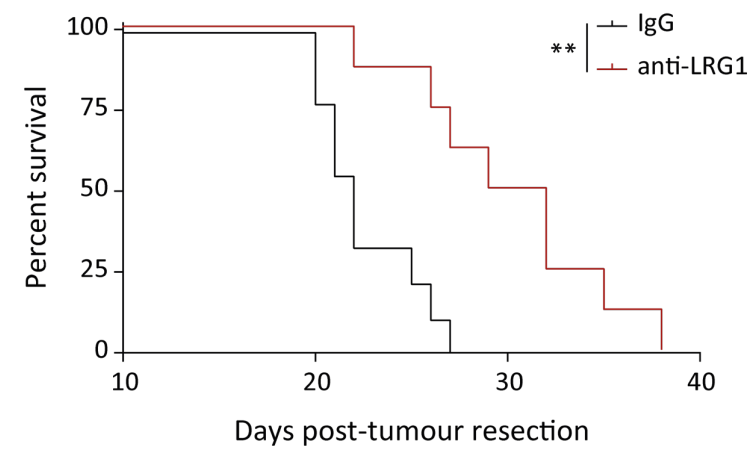


Fig. 4| LRG1 neutralization inhibits metastasis. a, Lrg1-overexpressing LLC (LLC-Lrg1) or control-LLC (LLC-pLenti) cells were subcutaneously inoculated in mice. 7 days later, melanoma (B16F10) cells were intravenously injected. On the left, dot plot showing the number of melanoma metastases in the lung, and on the right, representative lung images (mean $\pm S D, n=12$ mice). Scale bars $=5 \mathrm{~mm}$. *, $\mathrm{P}<0.05$ (two-tailed Mann-Whitney $U$ test). b-e, WT or NG2-Cre X YFP fl/fl mice were injected with either Lrg1-overexpressing LLC (LLC-Lrg1) or control-LLC (LLC-pLenti) cells. FACS-based quantitation of EC, immune cells, and $\mathrm{NG}^{+}$perivascular cells in the lung of tumour-bearing mice (b, c) (mean \pm $\mathrm{SD}, \mathrm{n}=5-6$ mice). ${ }^{* *}, \mathrm{P}<0.01$ (two-tailed Mann-Whitney $U$ test). Lung tissue sections were stained for Desmin (pericyte-specific) and $\alpha \mathrm{SMA}$ (smooth muscle cell-specific). Representative images of lung sections (d). Scale bars $=100 \mu \mathrm{m}$. Quantitation of Desmin/DAPI area and aSMA/DAPI area are shown (e) (mean $\pm S D, n=10$ mice; two-tailed Mann-Whitney $U$ test). $f-h$, Therapeutic assessment of LRG1-blocking antibody 15C4 in LLC metastasis model using two different strategies (f). KaplanMeier graphs showing overall survival of mice after primary tumour resection when treated with control-IgG or anti-LRG1 in perioperative ( $g ; n=10$ mice) or postsurgical adjuvant $(h ; n=8-9$ mice) setting (50 mg/kg twice per week). *, $\mathrm{P}<0.05 ;{ }^{* *}, \mathrm{P}<0.01$ (Log-rank (Mantel-Cox) test). 